Modern Physics Letters A,

(C) World Scientific Publishing Company

\title{
Causal Propagation of Spin-Cascades
}

\author{
L. M. RICO, M. KIRCHBACH * $^{*}$ \\ Instituto de Física, Universidad Aut. de San Luis Potosí, \\ Av. Manuel Nava 6, Zona Universitaria, \\ San Luis Potosí, SLP 78290, México \\ Received (received date) \\ Revised (revised date)
}

\begin{abstract}
We gauge the direct product of the Proca with the Dirac equation that describes the coupling to the electromagnetic field of the spin-cascade $(1 / 2,3 / 2)$ residing in the four-vector spinor $\psi_{\mu}$ and analyze propagation of its wave fronts in terms of the Courant-Hilbert criteria. We show that the differential equation under consideration is unconditionally hyperbolic and the propagation of its wave fronts unconditionally causal. In this way we proof that the irreducible spin-cascade embedded within $\psi_{\mu}$ is free from the Velo-Zwanziger problem that plagues the Rarita-Schwinger description of spin-3/2. The proof extends also to the direct product of two Proca equations and implies causal propagation of the spin-cascade $(0,1,2)$ within an electromagnetic environment.
\end{abstract}

Keywords: High-spins; causal propagation.

\section{Introduction}

The consistent description of high-spins within a covariant framework is a long standing problem in particle physics. The commonly used description of fermions with spin higher than $1 / 2$ takes its origin from Refs. 1,2 which suggested to view fractional spin $J=(K+1 / 2)$ with $K$ integer as the highest spin in the totally symmetric rank $-K$ tensor spinor $\psi_{\mu_{1} \mu_{2} \ldots \mu_{K}}$ and describe it by means of the Dirac equation,

$$
(\not p-m) \psi_{\mu_{1} \mu_{2} \ldots \mu_{K}}=0 \text {, }
$$

*E-mail: mariana@ifisica.uaslp.mx 
as supplemented by the two auxiliary conditions

$$
\begin{aligned}
& p^{\mu_{1}} \psi_{\mu_{1} \mu_{2} \ldots \mu_{K}}=0, \\
& \gamma^{\mu_{1}} \psi_{\mu_{1} \mu_{2} \ldots \mu_{K}}=0,
\end{aligned}
$$

with $p^{\mu}$ being the four momentum. In so doing one restricts the degrees of freedom to $2(2 J+1)$ which are then associated with spin- $J$ particles and antiparticles. The tensor-spinor representation spaces reside in the direct products of $K$ four-vector copies with the Dirac spinor $\psi=(1 / 2,0) \oplus(0,1 / 2)$ according to

$$
\begin{aligned}
\psi_{\mu_{1} \mu_{2} \ldots \mu_{K}}= & \operatorname{Sym}(1 / 2,1 / 2)_{1} \otimes(1 / 2,1 / 2)_{2} \otimes \ldots \otimes(1 / 2,1 / 2)_{K} \\
& \otimes[(1 / 2,0) \oplus(0,1 / 2)] .
\end{aligned}
$$

They consist in their rest frames of $K$ parity doublets with spins ranging from $1 / 2^{ \pm}$ to $(K-1 / 2)^{ \pm}$while the highest spin $J=K+1 / 2$ remains a parity singlet:

$$
\psi_{\mu_{1} \mu_{2} \ldots \mu_{K}} \stackrel{\text { rest frame }}{\longrightarrow} \frac{1^{+}}{2}, \frac{1}{2}^{-} ; \frac{3}{2}^{+}, \frac{3}{2}^{-} ; \ldots ;\left(K-\frac{1}{2}\right)^{+},\left(K-\frac{1}{2}\right)^{-} ;\left(K+\frac{1}{2}\right)^{\pi} .
$$

Here the parity of the highest spin is $(-1)^{K}$ for tensors, and $(-1)^{K+1}$ for pseudotensors. Equation (5) illustrates the meaning of $\psi_{\mu_{1} \mu_{2} \ldots \mu_{K}}$ as spin-cascades. The tensor-spinor of lowest rank is the four-vector spinor, $\psi_{\mu}$. It is used in the description of spin-3/2, in which case one encounters the shortest spin-cascade

$$
\psi_{\mu} \stackrel{\text { rest frame }}{\longrightarrow} \frac{1}{2}^{+}, \frac{1}{2}^{-} ; \frac{3}{2}^{-}
$$

Here we choose the polar four-vector spinor for concreteness. Applied to $\psi_{\mu}$, Eqs. (1)-(3) reduce the 16 degrees of freedom to 8 and associate them with particles and anti-particles of spin-3/2 at rest. The first auxiliary condition in Eq. (2) excludes the spin- $0^{+}$component of the four-vector and thereby the spin- $1 / 2^{+}$part of $\psi_{\mu}$, while the second auxiliary condition in Eq. (3) excludes its parity counterpart spin- $1 / 2^{-}$. The tensor-spinor framework can be given a Lagrangian formulation upon establishing the most general form of a Lagrangian that leads to the above three equations. Such a Lagrangian for the four-vector-spinor $\psi_{\mu}$ can be found in 3 and reads

$$
L(A)=\bar{\psi}^{\mu}\left[p_{\alpha} \Gamma_{\mu \nu}^{\alpha}-m g_{\mu \nu}\right] \psi^{\nu}
$$

where

$$
\begin{gathered}
p_{\alpha} \Gamma_{\mu \nu}^{\alpha}(A) \psi^{\nu}=\not p \psi_{\mu}+B(A) \gamma_{\mu} \not p \gamma \cdot \psi+A\left(\gamma_{\mu} p \cdot \psi+p_{\mu} \gamma \cdot \psi\right)+C(A) m \gamma_{\mu} \gamma \cdot \psi, \\
A \neq \frac{1}{2}, \quad B(A) \equiv \frac{3}{2} A^{2}+A+\frac{1}{2}, C(A)=3 A^{2}+3 A+1 .
\end{gathered}
$$

The wave equation following from the above Lagrangian is obtained as

$$
(\not p-m) \psi_{\mu}+A\left(\gamma_{\mu} p \cdot \psi+p_{\mu} \gamma \cdot \psi\right)+B(A)\left(\gamma_{\mu} \not p \gamma \cdot \psi\right)+C(A) m \gamma_{\mu} \gamma \cdot \psi=0
$$


which for $A=-1$ can be written in a compact form as

$$
\left(i \varepsilon_{\mu \nu \beta \alpha} \gamma^{5} \gamma^{\beta} p^{\alpha}-m g_{\mu \nu}+m \gamma_{\mu} \gamma_{\nu}\right) \psi^{\nu}=0 .
$$

Equations of this type are equivalent to

$$
\begin{aligned}
\frac{1}{2 m}(\not p+m) \psi_{\mu} & =\psi_{\mu}, \\
\left(-g_{\mu \nu}+\frac{1}{m^{2}} p_{\mu} p_{\nu}\right) \psi^{\nu} & =-\psi_{\mu}, \\
\gamma^{\mu} \psi_{\mu} & =0,
\end{aligned}
$$

known as the Rarita-Schwinger (RS) framework ${ }^{2}$ Notice that for the sake of convenience of the point we are going to make in the next section, we here wrote the respective Dirac and Proca equations (11), and (12) in terms of covariant projectors picking up spin- $1 / 2^{+}$and spin- $1^{-}$states, respectively. Spin $3 / 2^{+}$needs an axial four vector. Equation (11)-(13) suffer several inconsistencies one of them being the non-causal propagation of the classical wave fronts of its solutions, a result due to Ref ${ }^{4}$ and known as the Velo-Zwanziger problem. Furthermore, the inverse of Eq. (9) does not relate to the spin $3 / 2$ projector alone but is a more complicated combination of various projectors $\underline{5}$. This inconvenience can affect the quantization procedure which is also known to suffer inconsistencies 6 .

We here made the case that the Velo-Zwanziger problem is not inherent to the $\psi_{\mu}$ representation space by itself but rather to its description within the Rarita-Schwinger framework. To be specific, we shall show that the propagation of the spin cascade $(1 / 2,3 / 2)$ when described in terms of the direct product of the Proca and Dirac equations does not suffer the Velo-Zwanziger problem but propagates strictly causally. In addition, one gains coincidence between the nominator in the propagator (the latter being the inverse of the wave equation) and the projector built from the states.

Spin-cascade propagations are of interest in the physics of baryon resonances where according to Ref. 7 (and references therein) one observes well pronounced massand parity degeneracies patterned after the tensor-spinors of rank-1, 3, and 5, respectively, but also possibly for the physics of the gravitino and the graviton, an idea that has been put forward in Refs. 8,9 . The idea of using spin-cascades as gauge fields in unified theories has been pioneered by Kruglov and collaborators (see Ref. 10 and references there in).

The paper is organized as follows. In the next section we briefly review the essentials of the Velo-Zwanziger problem of acuasal spin-3/2 propagation in the light of the Currant-Hilbert criteria. Section III is devoted to the propagation properties of the spin-cascades $(1 / 2,3 / 2)$ and $(0,1,2)$. The paper ends with a brief summary. 


\section{The Velo-Zwanziger problem of high-spin propagation}

The non-causal propagation of spin-3/2 within the gauged Rarita-Schwinger framework has first been addressed in the work of Giorgio Velo and Daniel Zwanziger ${ }^{4}$. For the sake of self sufficiency of the presentation we here highlight it in brief. The main point of Ref. 4 is that Eq. (10) provided by the Lagrangian (8) is not a genuine first order equation of motion because it does not contain any time derivative of $\psi_{0}$ at all.

This defect shows up in the (i) complete cancellation of all $\partial_{0} \psi_{0}$ terms in Eq. (10) for any $\mu$, (ii) complete cancellation of all the $\partial_{0} \psi_{\alpha}$ terms for $\mu=0$, in which case one finds instead of a wave equation the constraint

$$
[\mathbf{p}+(\mathbf{p} \cdot \gamma-m) \gamma] \cdot \psi=0,
$$

(iii) absence of $\psi_{0}$ in Eq. (14) that leaves the time-component of the RaritaSchwinger field undetermined. The above deficits are caused by the constraints hidden in the wave equation and could be tolerated only if remediable upon gauging. Velo and Zwanziger gauge Eq. (10) in Ref. 4 in replacing $p_{\mu}$ by $\pi_{\mu}=p_{\mu}+e A_{\mu}$, and succeed in constructing a genuine equation. Their remedy procedure begins with first contracting the gauged equation successively by $\gamma^{\mu}$ and $\pi^{\mu}$ and obtaining the covariant gauged constraints as

$$
\begin{aligned}
& \gamma \cdot \psi=-\frac{2}{3} \frac{i e}{m^{2}} \gamma^{5} \gamma \cdot \widetilde{F} \cdot \psi \\
& \pi \cdot \psi=-\left(\gamma \cdot \pi+\frac{3}{2} m\right) \frac{2}{3} \frac{i e}{m^{2}} \gamma^{5} \gamma \cdot \widetilde{F} \cdot \psi,
\end{aligned}
$$

and ends with substituting Eqs. (15]16) back into the gauged Eq. (10). The resulting new wave equation,

$$
(\AA-m) \psi_{\mu}+\left(\pi_{\mu}+\frac{m}{2} \gamma_{\mu}\right) \frac{2}{3} \frac{i e}{m^{2}} \gamma^{5} \gamma \cdot \widetilde{F} \cdot \psi=0,
$$

is now a genuine one because it can be shown to determine both $\psi_{0}$ and the time derivatives of $\psi_{\mu}$ for any given $\mu$.

The final goal is to test hyperbolicity and causality of Eq. (17) by means of the Courant-Hilbert criterion 11 which requires the so called characteristic determinant of the matrix containing the highest derivatives when replaced by $n_{\mu}$, i.e. by the normals to the characteristic surfaces, to vanish only for real $n_{0}$. The CourantHilbert criterion is applied in fact not directly to Eq. (17) but to its Hermitian form as obtained by using repeatedly Eqs. (15) and (16):

$$
\begin{aligned}
(\gamma \cdot \pi-m) \psi_{\mu}+\left(\pi_{\mu}\right. & \left.+\frac{1}{2} m \gamma_{\mu}\right) \frac{2 i e}{3 m^{2}} \gamma^{5} \gamma \cdot \widetilde{F} \cdot \psi \\
& +\frac{2 i e}{3 m^{2}} \widetilde{F}_{\mu} \cdot \gamma \gamma^{5}\left(\pi+\frac{1}{2} m \gamma\right) \cdot \psi \\
& +\frac{2 i e}{3 m^{2}} \widetilde{F}_{\mu} \cdot \gamma \gamma^{5}(\gamma \cdot \pi+2 m) \frac{2 i e}{3 m^{2}} \gamma^{5} \gamma \cdot \widetilde{F} \cdot \psi=0
\end{aligned}
$$


The last equation represents now a system of partial differential equations which would describe wave propagation phenomena provided it were hyperbolic, something that can be tested by exploiting the Courant-Hilbert criterion. For this purpose it is sufficient to compute the normals $n_{\mu}$ to the characteristic surfaces. To find the normals to the characteristic surfaces passing through each point we replace $i \partial_{\mu}$ by $n_{\mu}$ in the highest derivatives and calculate the determinant $D(n)$ of the resulting coefficient matrix, which is called the characteristic determinant. The equation of motion Eq. (18) will be hyperbolic if the solutions $n^{0}$ to $D(n)=0$ are real for any $n_{\mu}=\left(n^{0}, \mathbf{n}\right)$. By means of this prescription we are left with the following characteristic determinant for the Rarita-Schwinger framework,

$$
\begin{aligned}
D(n) & =\mid \gamma \cdot n g_{\mu}{ }^{\nu}+\frac{2 i e}{3 m^{2}} n_{\mu} \gamma^{5} \gamma \cdot \widetilde{F}^{\nu}+\frac{2 i e}{3 m^{2}} \widetilde{F}_{\mu} \cdot \gamma \gamma^{5} n^{\nu} \\
& +\left(\frac{2 i e}{3 m^{2}}\right)^{2} \widetilde{F}_{\mu} \cdot \gamma \gamma^{5}(\gamma \cdot n) \gamma^{5} \gamma \cdot \widetilde{F}^{\nu} \mid
\end{aligned}
$$

The covariant form of the latter is found to be,

$$
D(n)=\left(n^{2}\right)^{4}\left[n^{2}+\left(\frac{2 e}{3 m^{2}}\right)^{2}(\widetilde{F} \cdot n)^{2}\right]^{4}=0 .
$$

Equation (20) has the following four positive and four negative roots

$$
n_{0}= \pm \sqrt{\mathbf{n}^{2}}
$$

Eight more roots are found from

$$
n^{0}= \pm \sqrt{\frac{\left(\mathbf{n}^{2}-k^{2}(\mathbf{B} \cdot \mathbf{n})^{2}\right)}{1-k^{2} \mathbf{B}^{2}}}= \pm \frac{\sqrt{\mathbf{n}^{2}\left(1-k^{2} \mathbf{B}^{2} \cos ^{2} \lambda\right)\left(1-k^{2} \mathbf{B}^{2}\right)}}{1-k^{2} \mathbf{B}^{2}}
$$

respectively, where $\mathbf{B}$ stands for the magnetic field, $k=\frac{2 e}{3 m^{2}}$, and $\lambda$ is the angle between $\mathbf{B}$ and $\mathbf{n}$. Introducing the notion of the "weak-field limit" to refer to the situation in which there exists, for each space-time point, a Lorentz frame such that the inequality,

$$
k^{2} \mathbf{B}^{2} \leq 1,
$$

is satisfied, we see that the roots given by Eq. (22) are real for any given $n=\left(n^{0}, \mathbf{n}\right)$, which establishes hyperbolicity.

In the strong-field limit when the inequality (23) is no longer satisfied, Eq. (18) ceases to be hyperbolic and is not suitable for the description of wave phenomena at all.

Moreover, in recalling that the maximum velocity of the signal propagation is the slope of the characteristic surfaces, one immediately realizes that the characteristic surfaces determined by Eq. (20) are not all tangent to the light cone. Stated differently, one finds space-like characteristic surfaces passing through all points 
where $F_{\mu \nu}$ is non-vanishing. Consequently, such signals are propagated at velocities greater than the speed of light. To see this it is just a matter of realizing that there are time-like normals $n_{\mu}$ which satisfy the characteristic equation (20). Thus, Eq. (18) has characteristic surfaces which propagate non-causally. In fact, for $n_{\mu}=(1,0,0,0)$ Eq. (20) takes the form of equality,

$$
1=k^{2} \mathbf{B}^{2}
$$

and whenever $F_{\mu \nu} \neq 0$, there exists a Lorentz frame where the latter equation holds valid. The above considerations show that causal propagation requires unconditionally hyperbolic wave equations.

So far only few authors have proposed solutions to the Velo-Zwanziger problem of the linear Rarita-Schwinger Lagrangian 12!13 In Ref. 12 Rañada and Sierra elaborate a method which is equivalent to the on-shell Rarita-Schwinger framework but in their gauged formalism the two auxiliary conditions have been replaced by one differential equation. In effect, the number of degrees of freedom increases from eight to twelve and the theory in Ref. 12 describes spin- $1 / 2$ and spin- $3 / 2$ particles of different masses. In applying the Courant-Hilbert criterion to their wave equation, the authors of Ref. 12 prove that the wave fronts of the solutions of their equation indeed do propagate causally. More recently, Kruglov suggested two different wave equations, the first of which is of second order and non-local while the second is local and linear. 13 None of the above equations suffers the pathology of non-causal propagation of spin-3/2. The non-local equation has twelve degrees of freedom of equal masses which are associated with spin- $3 / 2$ and spin- $1 / 2$. The local equation uses a twenty dimensional space to embed the four-vector spinor which contains next to spin- $3 / 2$ also the two copies of spin- $1 / 2$ of opposite parities. Within the latter scenario the three different spin sectors of $\psi_{\mu}$ appear characterized by three different masses. Apparently, the mass-splittings between the spins is the price to be paid for the causal propagation of the solutions to linear wave equations. Finally, single spins of the type $(s, 0) \oplus(0, s)$ have been shown to propagate always causally, results due to Hurley 14 , and more recently to Ahluwalia and Ernst 15 On the one side, Hurley's focus is on the manifestly hyperbolic nature of the generalized Feynman-Gell-Mann equations for $(s, 0) \oplus(0, s)$,

$$
\left(\pi^{2}-m^{2}\right) \Psi^{(s, 0) \oplus(0, s)}=\frac{e}{2 s} S^{\mu \nu} F_{\mu \nu} \Psi^{(s, 0) \oplus(0, s)},
$$

which are of second order in the momenta, and obviously manifestly hyperbolic. The solutions of the generalized Feynman-Gell-Mann equations therefore propagate unconditionally causally. Ahluwalia and Ernst construct the $(s, 0) \oplus(0, s)$ propagation from the different perspective of the representation space and obtain it as third order in the momenta. Their causality proof is based on the correct energy-momentum dispersion relations. Despite their merits, the $(s, 0) \oplus(0, s)$ representations are not as popular because they are difficult to couple to the pion-nucleon or photon-nucleon 
system due to dimensionality mismatch, a reason that still represents an obstacle to their application in phenomenology.

In the next section we (i) consider as a new option a local but third order singlemass wave equation describing the $(1 / 2,3 / 2)$ cascade residing in $\psi_{\mu}$ and, (ii) deliver the proof of the causal propagation of its wave fronts within an electromagnetic environment. The major appeal of the latter option is that it matches well with the observed mass degeneracy of spin- $1 / 2^{-}$and $3 / 2^{-}$baryons such like, say, the $S_{11}(1535)$ and $D_{13}(1520)$ resonances 7 etc. From that perspective it is desirable to have a spin-cascade equation that is characterized by a single mass.

\section{Cascade--spin description and causal propagation}

In order to find the wave equation for the spin-cascade of interest it is quite instructive to go back to Eq. (44) and to recall the wave equation for the four-vector spinor. Although one encounters various equations for the $(1 / 2,1 / 2)$ representation space in the literature, 16,17, 18,19 all they reflect different facets of the following equation and related auxiliary condition:

$$
\begin{aligned}
{\left[\left(p^{2}-m^{2}\right)[g]_{\mu}^{\nu}-p_{\mu} p^{\nu}\right] A^{\mu} } & =0 \\
p_{\mu} A^{\mu} & =0 .
\end{aligned}
$$

The latter equations imply that the four degrees of freedom of the $\left(0^{+}, 1^{-}\right)$spin cascade in $(1 / 2,1 / 2)$ have been reduced to three and are associated with spin- 1 at rest. Now we consider the direct product of Eq. (27) with the Dirac equation

$$
(\not p-m) \psi=0,
$$

which will describe accordingly the spin-cascade $\left(1 / 2^{-}, 3 / 2^{-}\right)$,

$$
\begin{aligned}
{\left[\left(p^{2}-m^{2}\right)[g]_{\mu}^{\nu}-p_{\mu} p^{\nu}\right] \otimes(\not p-m) \psi^{\mu} } & =0, \\
\psi^{\mu} & =A^{\mu} \otimes \psi .
\end{aligned}
$$

The inverse of the latter equation has the advantage to provide a propagator,

$$
\Pi_{(1 / 2,3 / 2)}(p)=\frac{1}{2 m} \frac{\left(-g_{\mu \nu}+\frac{1}{m^{2}} p_{\mu} p_{\nu}\right)(\not p+m)}{p^{2}-m^{2}+i \epsilon},
$$

which is consistent with the Proca and Dirac projectors in Eqs. (12) and (11), respectively.

Upon gauging, Eq. (26) becomes

$$
\left[\left(\pi^{2}-m^{2}\right)[g]_{\mu}^{\nu}-\pi_{\mu} \pi^{\nu}\right](\pi-m) \psi^{\mu}=0,
$$

where we dropped the $\otimes$ sign for simplicity. If now one calculates the characteristic determinant $\left|\Gamma_{\nu \mu}\right|$ of the matrix that has as elements all the highest derivative terms with the derivatives being replaced by $n_{\mu}$, one finds it to be zero. This means that 
the latter equation has constraints built in which prevent it from being a genuine system of differential equations. This deficit is removed upon finding the gauged auxiliary conditions and substituting them back into the leading equation.

To do so we contract Eq. (31) by $\pi_{\nu}$ with the result,

$$
\begin{aligned}
{\left[\pi_{\mu}\left(\pi^{2}-m^{2}\right)-\left(\pi_{\mu} \pi_{\nu}+i e F_{\nu \mu}\right) \pi^{\nu}\right](\pi-m) \psi^{\mu} } & =0 \\
{\left[m^{2} \pi_{\mu}+i e F_{\nu \mu} \pi^{\nu}\right](\pi-m) \psi^{\mu} } & =0 \\
m^{2} \pi_{\mu}(\pi-m) \psi^{\mu} & =i e F_{\mu \lambda} \pi^{\lambda}(\pi-m) \psi^{\mu} .
\end{aligned}
$$

In order to incorporate the gauged auxiliary condition in Eqs. (32) into the wave equation we commute the canonical momenta to obtain $\pi_{\mu} \pi^{\nu}$ in Eq. (31),

$$
\begin{aligned}
{\left[\left(\pi^{2}-m^{2}\right)[g]_{\mu}^{\nu}-\left(\pi^{\nu} \pi_{\mu}+i e F_{\mu}{ }^{\nu}\right)\right](\pi-m) \psi^{\mu} } & =0, \\
\left\{\left(\pi^{2}-m^{2}\right)[g]^{\nu}{ }_{\mu}(\pi-m)-\pi^{\nu} \pi_{\mu}(\pi-m)+i e F^{\nu}{ }_{\mu}(\pi-m)\right\} \psi^{\mu} & =0, \\
\left\{\left(\pi^{2}-m^{2}\right)[g]^{\nu}{ }_{\mu}-\frac{i e}{m^{2}} \pi^{\nu} F_{\mu \lambda} \pi^{\lambda}+i e{F^{\nu}}_{\mu}\right\}(\pi-m) \psi^{\mu} & =0 .
\end{aligned}
$$

The following terms contribute to the characteristic determinant $\left|\Gamma_{\mu}^{\nu}\right|$ :

$$
\pi^{2} \Uparrow[g]_{\mu}^{\nu}+\frac{i e}{m^{2}} \pi^{\nu} F_{\lambda \mu} \pi^{\lambda} \Uparrow \rightarrow \Gamma_{\mu}^{\nu}=n^{2} \not h[g]_{\mu}^{\nu}+n^{\nu} \frac{i e}{m^{2}} F_{\lambda \mu} n^{\lambda} \not h .
$$

The determinant to be calculated can be cast into the form

$$
\left|\Gamma_{\mu}^{\nu}\right|=\left|n^{2} \not h[g]_{\mu}^{\nu}+n^{\nu} \frac{i e}{m^{2}} F_{\lambda \mu} n^{\lambda} \not h\right|=\left|\left(n^{2}[g]_{\mu}^{\nu}+n^{\nu} \frac{i e}{m^{2}} F_{\lambda \mu} n^{\lambda}\right) \otimes(\not h)\right| .
$$

The latter equation is no more but the direct product of $\left[\Gamma^{\nu}{ }_{\mu}\right]_{\text {Proca }}$ with $\left[\Gamma^{\nu}{ }_{\mu}\right]_{\text {Dirac }}$. We now can use the general theorem on the determinant of the direct (Kronecker) product of two matrices, here denoted by $A$, and $B$, of dimensionality $n$ and $q$, respectively, which tells one that

$$
|A \otimes B|=|A|^{q}|B|^{n} .
$$

In applying this formula to the direct product of

$$
\left[\Gamma_{\mu}^{\nu}\right]_{\text {Proca }}=\left(n^{2}\right)^{4}, \quad \text { and } \quad\left[\Gamma_{\mu}^{\nu}\right]_{\text {Dirac }}=\left(n^{2}\right)^{2},
$$

under consideration, we here find

$$
\left[\Gamma_{\mu}^{\nu}\right]_{\text {Proca } \otimes \text { Dirac }}=\left(n^{2}\right)^{24},
$$

and arrive at the satisfactory result on its unconditional hyperbolicity.

This result can be independently confirmed by using appropriate routines in symbolic mathematical codes such as Maple in the calculation of the $\left|\Gamma_{\mu}^{\nu}\right|$ determinant as

$$
\left|\Gamma_{\mu}^{\nu}\right|=\left|n^{2} \not h[g]_{\mu}^{\nu}+n^{\nu} \frac{i e}{m^{2}} F_{\lambda \mu} n^{\lambda} \not h\right|=\left(n^{2}\right)^{24} .
$$


The conclusion is that spin-cascades are allowed to propagate causally within the electromagnetic environment. Same is valid for the Kronecker products of arbitrary (finite) number of Proca equations on the basis of Eq. (36). An interesting case is the one of two Proca equations where one encounter the spin-cascade $(0,1,2)$, a case that may be of interest to gravity ${ }^{9}$ Also this cascade will propagate causally within the electromagnetic background, something which is out of reach for the $\psi_{\mu_{1} \mu_{2}}$ sector characterized by a single spin- 2 at rest, a result due to. 19

\section{Summary}

To summarize, we here found that Kronecker products of a finite number of Proca equations with or without the Dirac equation allow for causal propagation of the wave fronts of the associated solutions. Such solutions, in carrying factorized Lorentz and spinor indices are naturally coupled to the pion-nucleon, or photonnucleon systems and describe inseparable spin-cascades. The corresponding wave equations provide propagators that are consistent with the projectors onto the states and we expect this feature to facilitate the quantization procedure. Spin cascades such like $(1 / 2,3 / 2)$ and $(0,1,2)$ may be of interest both to particle spectroscopy and gravity.

\section{Acknowledgments}

We appreciate insightful discussions with Mauro Napsuciale on causality in the light of the Courant-Hilbert criterion.

Work supported by Consejo Nacional de Ciencia y Tecnología (CONACyT, Mexico) under grant number C01-39820.

\section{References}

1. M. Fierz, W. Pauli, Proc. Roy. Soc. (London) A173, 211 (1939).

2. W. Rarita, J. Schwinger, Phys. Rev. 60, 61 (1941).

3. P. A. Moldauer, K.M. Case, Phys Rev. 102, 279 (1956).

4. G. Velo, D. Zwanziger, Phys. Rev. 186, 1337 (1969).

5. J. Weda, Spin-3/2 particles and consistent $\pi N \Delta$ and $\gamma N \Delta$-couplings, Ph. D. thesis, KVI, University of Croningen, July, 1999.

6. K. Johnson, E. C. Sudarshan, Annals of Physics 13, 126 (1961).

7. M. Kirchbach, M. Moshinsky, Yu. F. Smirnov, Phys. Rev. D64, 114005 (2001).

8. M. Kirchbach, D. V. Ahluwalia, Phys. Lett. B529, 124 (2002).

9. D. V. Ahluwalia, N. Dadhich, M. Kirchbach, Int. J. Mod. Phys. D 11, 1621 (2002).

10. S. I. Kruglov, Symmetry and Electromagnetic Interactions of Fields with MultiSpin (Nova Science Publishers, Huntington, N.Y. 2001) p. 216.

11. R. Courant, D. Hilbert, Methods of Mathematical Physics (Wiley Inter-science, New York, 1962).

12. A. F. Rañada, G. Sierra, Phys. Rev. D22, 2416 (1980).

13. S. I. Kruglov, Int. J. Mod. Phys. A21, 1143 (2006).

14. W. J. Hurley, Phys. Rev. D4, 3605 (1971).

15. D. V. Ahluwalia, D. J. Ernst, Int. J. Mod. Phys. E2, 397 (1996). 
16. L. H. Ryder, Quantum Field Theory (Cambridge University Press, 1985)

17. D. V. Ahluwalia, M. Kirchbach, Mod. Phys. Lett. A16, 1377 (2001).

18. M. Napsuciale, C. A. Vaquera-Araujo, Equations of motion as projectors and the gyromagnetic factor $g(s)=1 / s$ from first principles, hep-ph/0310106.

19. G. Velo, D. Zwanziger, Phys. Rev. 188, 2218 (1969). 